\title{
Interacting with Inter ACT
}

\author{
M. J. ACKERMAN \\ Behavioral Sciences Department, Naval Medical Research Institute, Bethesda, Maryland 20014
}

A patch for making ACT loadable by RDOS and a program that makes ACT program tapes compatible with editors available from the computer manufacturer are described. These two facilities allow greater ease in loading ACT and modifying ACT programs.

Several computer systems have been developed (Wood, Sette, \& Weiss, 1975) that relieve the behavioral scientist of the cumbersome chore of computer language and operating system development and allow him merely to write the logical sequence necessary to accomplish a given behavioral task. Most of the systems so far developed suffer from a common problem: They are basically process-control languages and offer little or no data-handling or computational capability

Naval Medical Research and Development Command, Research Task MR041.01.03-0148. The opinions and statements contained herein are private ones of the author and are not to be construed as official or reflecting the views of the Navy Department or the Naval service at large. The author wishes to express his thanks and gratitute to Mary M. Matzen and Doris N. Auer for their help in the preparation of this paper.
(Marriott \& Voigtman, 1976). These computational functions usually can be carried out under the operating system supplied by the computer manufacturer in either FORTRAN or BASIC. Yet, many of these behavioral systems are incompatible with the regular operating system of a computer, and, therefore, it is either difficult or impossible to utilize some of the resources of that system.

One such behhavioral language is ACT (Millenson, 1971), marketed by BRS/LVE as the Inter $A C T^{1}$ system. It is designed to run with any Data General Corporation computer, but is not compatible with Data General's operating system, RDOS, or any of its subsets, DOS, SOS, SOS-MT, SOS-CT, or supersets, MRDOS or AOS. Total compatibility requires a major rewrite of the ACT operating system, but partial compatibility, so that RDOS resources can be utilized, has been accomplished.

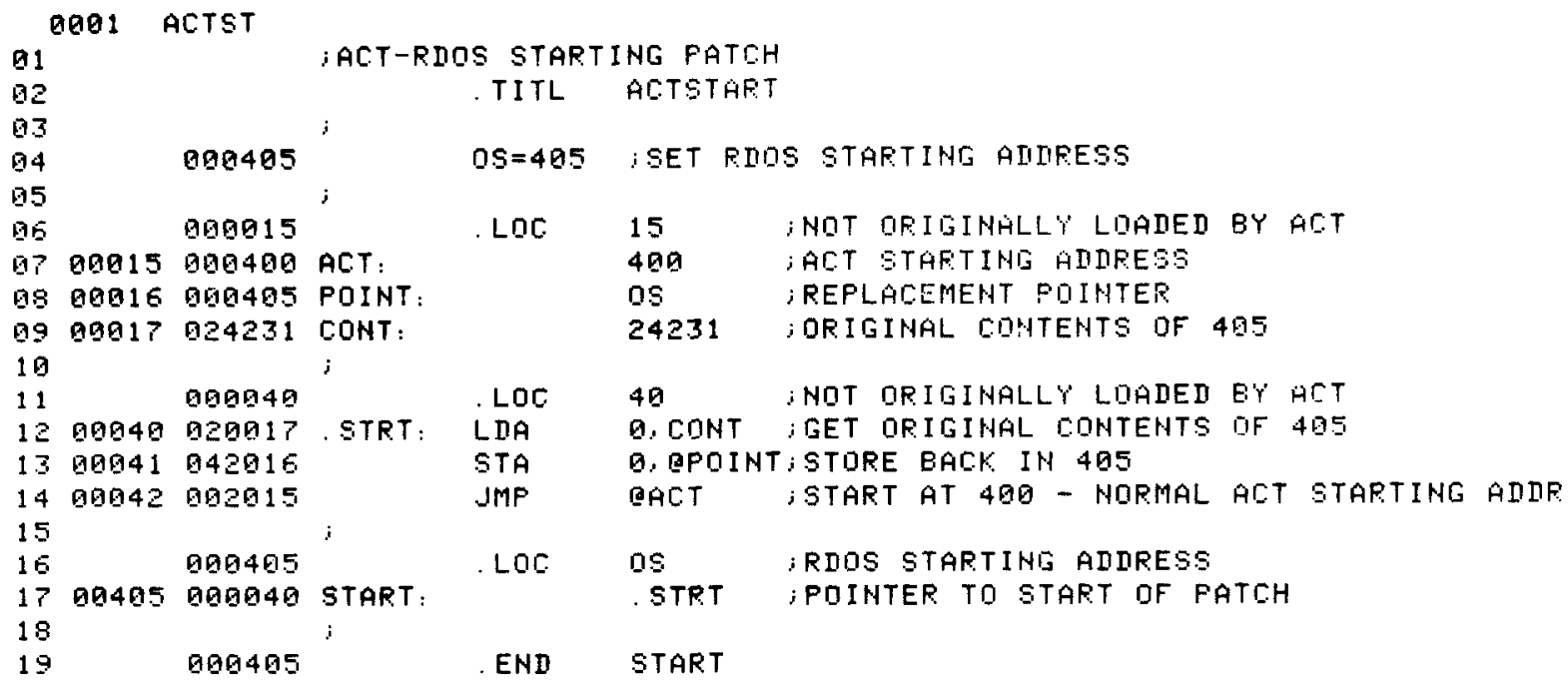

$\begin{array}{lllll}\quad 0092 & \text { ACTST } & & & \\ \text { ACT } & 000915 & 1 / 97 & 1 / 14 & \\ \text { CONT } & 090917 & 1 / 09 & 1 / 12 & \\ \text { OS } & 009495 & 1 / 94 & 1 / 08 & 1 / 16 \\ \text { FOINT } & 090916 & 1 / 09 & 1 / 13 & \\ \text { START } & 090495 & 1 / 17 & 1 / 19 & \\ \text { STRT } & 090949 & 1 / 12 & 1 / 17 & \end{array}$

Figure 1. ACT patch for direct loading of ACT by RDOS. 

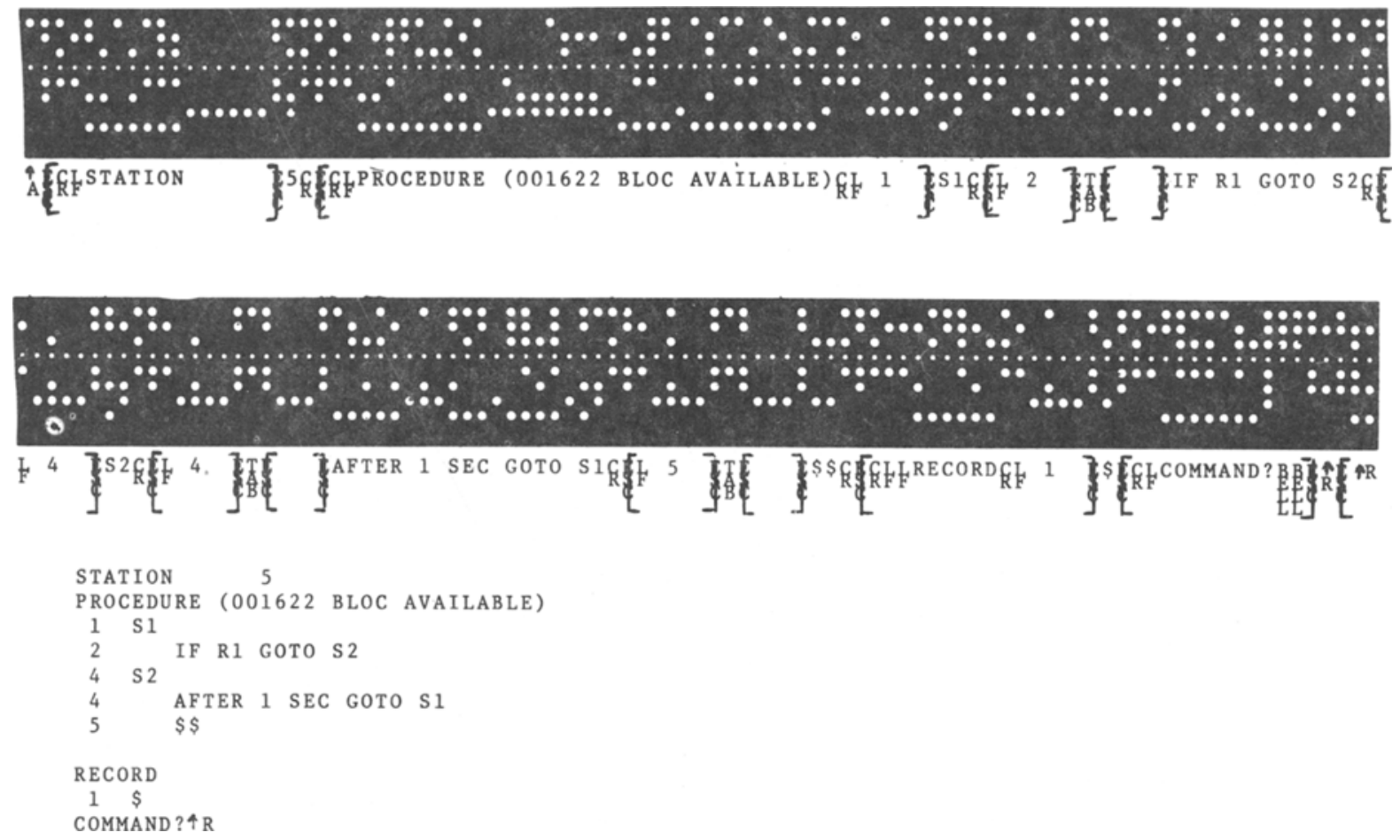

Figure 2. ACT program as output by ACT compiler.

\section{LOADING ACT VIA RDOS}

The ACT operating system cannot be loaded directly by RDOS because of an incompatibility in the starting address. The starting address in RDOS comes from memory Location 405. ACT uses this location as part of its program. The patch provided in Figure 1 corrects this incompatibility. If used with the stand-alone binary loader, the initial starting address is 40 . The clear and start address remains 400 and the restart address remains 426. This patch should be made part of the absolute binary ACT tape (Data General Corporation, Note 1) and binary punch utility. Care should be taken to punch the additional memory locations used by this patch. The patched version of ACT can be stored on magnetic tape or cassette with the MKSAVE command (SOS-MT or SOS-CT) or on disk with the MKSAVE/Z command (RDOS or DOS). Loading is accomplished via the MT-:- or CT-:- command (SOS-MT or SOS-CT) or via the BOOT-.-/A command (RDOS or DOS). Resetting ACT Location 426 to X7370, where X depends on available core, will protect the binary loader under SOS-MT or SOS-CT and only slightly decrease available memory.

\section{EDITING ACT PROGRAM TAPES}

Making changes in a behavioral program under the ACT operating system is theoretically simple because it involves only making changes in the logical sequence instructions. Nevertheless, the editing facility provided by the ACT operating system is extremely limited, which makes this simple task long and laborious. Data General provides several text editors under its RDOS (and subset) operating system, the simplest of which is EDIT. But program tapes punched by ACT cannot be read by EDIT because the parity bit is not punched, and because of an unfortunate choice of delimiter character. EDIT uses the ESCape character (ASCII 33 ${ }_{8}$ ) as its end-of-command line character; ACT uses the same character as its input switch character.

Under the ACT operating systems, programs are punched by the Teletype paper-tape punch while they are input. If an error is made, an error message is output to the Teletype printer and is also punched on the program paper tape. Certain Teletype control characters are necessary (i.e., line feed) to make the printer output readable; compiler prompts are given to elicit proper input. All of these extraneous characters are also punched on the program paper tape. A method of skipping the extraneous characters was devised so that the tapes could be used for future program input. A nonprintable character ESCape (ASCII 338) is automatically punched immediately before and immediately after such extraneous characters (Figure 2). Because it is a nonprintable character, it is transparent to the Teletype printer output. In effect, this character acts as a switch, telling the ACT compiler to skip all characters between the ESCape characters. Unfortunately, Data General's EDITor uses the same ESCape character as its command line-control character; therefore, 

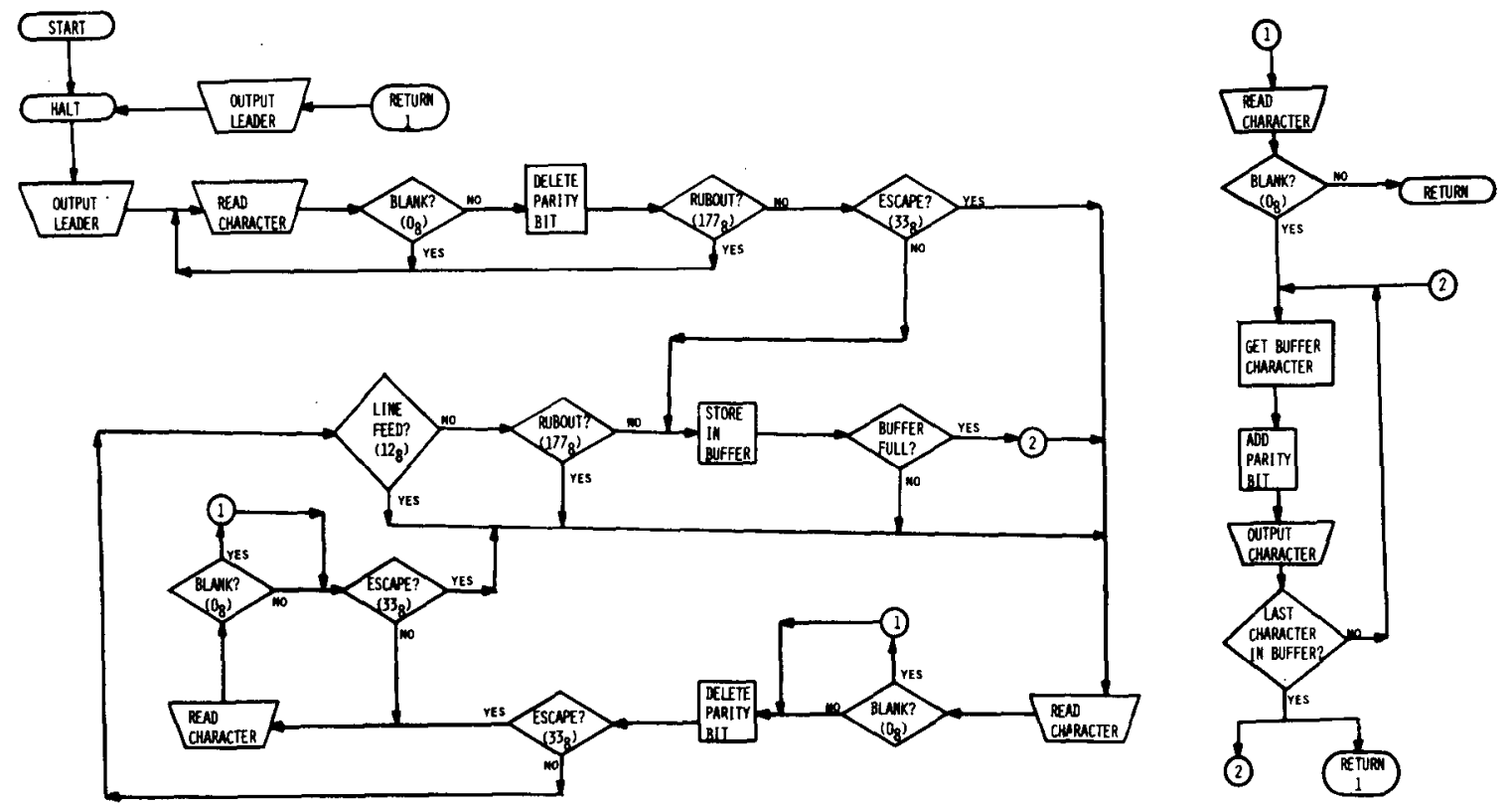

Figure 3. Flow chart of program to make ACT program tapes compatible with EDIT.

tapes punched by the ACT operating system are not compatible with EDIT. This problem can be corrected by substituting a different character at ACT Location 376 for the ESCape character. Control F (ASCII 78) has been suggested by BRS/LVE, but tapes using this character, or any other character, are incompatible with ACT program tapes and systems that use the ESCape character.

Another solution is to eliminate the switch character from program tapes. The short, stand-alone, flowcharted program in Figure 3 eliminates the switch character and all of the information in between. This not only makes ACT program tapes compatible with EDIT, but also greatly shortens the length of the tapes and maintains compatibility with other ACT systems (Figure 4). The program also adds the proper parity bits. The program as originally written was designed for high-speed paper-tape reader and punch, but it may be used with Teletype reader and punch by substituting TTI for PTR and/or TTO for PTP. Tapes generated by the program may be read directly by EDIT. Program tapes and listing are available from the author.

Tapes generated by EDIT may be read directly by ACT through the high-speed paper-tape reader. If Teletype input is used, the tapes must first be passed through the above program to eliminate the line-feed character (ASCII 128) that is generated by EDIT and not required by ACT. Otherwise, ERROR 1417, "illegal initial character," will be generated at the start of each line (Figure 5). This is merely an annoyance, because the program runs despite it.

The ability to use a full editor to make changes in ACT programs, combined with the ability to load ACT rapidly from magnetic tape, cassette, or disk, has made interacting with InterACT much more profitable, and certainly less frustrating, than was previously possible.

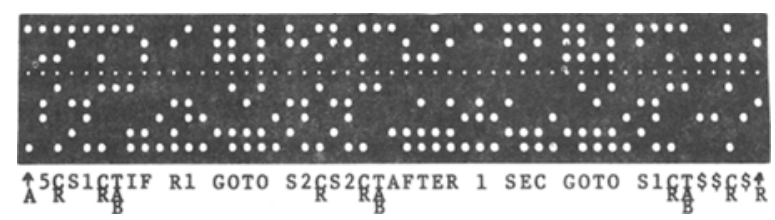

Figure 4. ACT program as output by ACT-EDTT compatibility program.
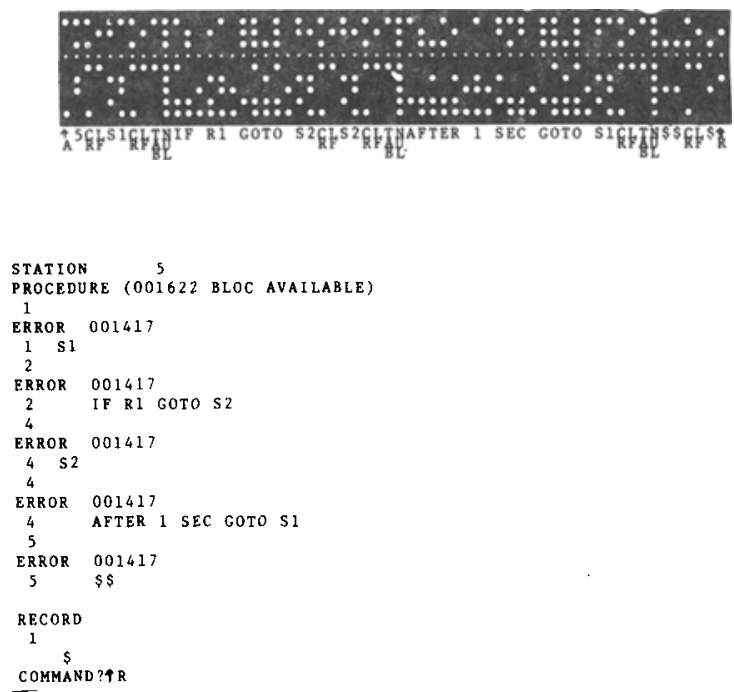

Figure 5. ACT program as output by EDIT, showing ignorable error message when input to ACT compiler. 


\section{REFERENCE NOTE}

1. Data General Corporation. How to use the Nova computers. Southboro, Mass: Data General Corporation, 1972.

\section{REFERENCES}

Marriott, J. G., \& Voigtman, R. E. An enchancement to ACT: A stand-alone disk driver compatible with RDOS. Behavior Research Methods \& Instrumentation, 1976, 8, 245-251.
Millenson, J. R. A programming language for on-line control of psychological experiments. Behavioral Science, $1971,16,248-256$.

Wood, R. W., Sette, W. F., \& Weiss, B. Interfacing the experimenter to the computer: Language for psychologists. American Psychologist, 1975, 30, 230-238.

\section{NOTE}

1. The InterACT system is available from the BRS/LVS division of the Tech Serv International, Inc., 5301 Holland Drive, Beltsville, Maryland 20705. 